\title{
An ETX Based Positioning System for Wireless Ad-hoc Networks
}

A. K. M. Mahtab Hossain, Preechai Mekbungwan, and Kanchana Kanchanasut

Internet Education and Research Laboratory (intERLab),

Asian Institute of Technology, Thailand.

\{mahtab, preechaim, Kanchana\}@ait.ac.th

\begin{abstract}
RF-based localization has gained popularity because it offers low-cost positioning solution for ad-hoc networks. The Received Signal Strength (RSS) measured by a node has traditionally been used as a parameter to estimate location. However, RSS is not made readily available in the ad-hoc routing protocols like some other link quality indication parameter, e.g., Expected Transmission Count (ETX). ETX predicts the number of transmissions required to deliver a packet over a particular link, including retransmissions. We reveal that ETX can be shown as a proximity indicator relative to an anchor node (i.e., node with known position), and thereby, could also be utilized as a location estimation parameter similar to RSS. We implement a localization plugin for the popular ad-hoc routing protocol, Optimized Link State Routing (OLSR) based on ETX. Our analysis and experiments show favorable results.
\end{abstract}

Key words: Localization, Positioning System, Ad-hoc Networks, Expected Transmission Count (ETX), Optimized Link State Routing (OLSR).

\section{Introduction}

Ad-hoc network is generally characterized by a large number of unattended inexpensive nodes with varying capabilities devoid of any fixed infrastructure [1]. The mass production of cheap and low power sensors is making ad-hoc networks more ubiquitous where the sensors are used in health-care, habitat monitoring, inventory tracking, battle field surveillance, disaster management and forecasting, etc [2]. Many of these applications require the knowledge of the positions of the nodes.

Radio positioning techniques support virtually any wireless device, integrate well with existing data networks, and operate both outdoors and indoors. The Received Signal Strength (RSS) perceived by the transceiver has traditionally been utilized in calculating the position of such wireless device. The RSS measurements between a node and the anchors (i.e., nodes with known locations) are generally converted into range (i.e., distance) estimates using either empirical or existing RF propagation models [3], and multi-lateration algorithms are applied. This approach of localization is called range-based. On the other hand, a rangefree scheme may discard the quantitative RSS values altogether. However, in this 
approach, the successful reception of radio messages from an anchor indicates whether the node is connected to that particular anchor or not (i.e., whether the node is close to the anchor in space). Subsequently, some connectivity-based algorithms like Centroid [4], APIT [5], etc. are applied for location estimation. Range-free schemes find more applicability in ad-hoc networks comprising of inexpensive nodes despite giving coarser accuracy. This is due to the fact that range-based schemes require complex hardware (e.g., directional antennae) in order to be accurate.

We aim to provide location as an add-on service for the wireless ad-hoc network that requires minimum or no modification at the node's protocol. The commercially accessible 802.11 (Wi-Fi) network interface cards (NICs) do not provide the RSS readings directly. Instead, a typical NIC only provides the Received Signal Strength Indicator (RSSI) parameter, in the form of an 8-bit unsigned integer which is intended for internal use by the NIC, e.g., to determine whether the channel is clear to send, or to decide whether it should attempt to roam. The 802.11 standard does not mandate how RSSI should be calculated from the sampled RSS. As a result, different vendors tend to have their own formulas or conversion tables for the mapping from RSS to RSSI, and vice versa [6]. Moreover, some NICs do not report RSS in user space of the operating system. The popular ad-hoc routing protocols (e.g., DSR [7], DSDV [8], OLSR [9], AODV [10], etc.) also do not make RSS available through their APIs. In order to integrate these protocols with location information, one has to make link layer system calls for retrieving the RSS values which may incur significant overhead for the protocol running on inexpensive sensors. Since we require a localization solution that could be transparently run over the various types of devices or sensors with little or no intervention, RSS option seems not too attractive.

The ad-hoc routing protocols may use some link quality metrics in order to discover efficient path. These metrics can easily be indicative of the connectivity of the node to its neighbors for the range-free algorithms of localization discussed previously. The better the link quality, the more connected the node is to its neighbor. Expected Transmission Count (ETX) is one such link quality indication parameter. ETX metric was first proposed in [11] to model the expected number of transmissions required to send a unicast packet over a link, including retransmissions. It is calculated by taking into account the successfully transmitted and received packets between a node and its neighbor within a certain time period. In this paper, we propose a positioning system for ad-hoc networks based on ETX. We analytically show ETX to be indicative of a node's connectivity or proximity to the anchors, and thereby, argue that it could be used to estimate its location. We also implement a localization plugin for the popular ad-hoc routing protocol, OLSR, based on ETX, and conduct experiments. Our experiments show favorable results which strengthen our claim that, ETX could be used as a location estimation parameter similar to RSS. We choose ETX as a location estimation parameter for ad-hoc networks for the following reasons: i) it is readily available as an extension to the popular ad-hoc routing protocols, e.g., DSR, DSDV, OLSR, etc [11,9], ii) it is an efficient proximity indication 
metric which will be elaborately discussed in Section 3.2, and iii) since it is a network layer metric, no interoperability issue across different NICs as discussed in case of RSS.

The rest of the paper is organized as follows. We provide a brief description of related works in Section 2. In Section 3, we present a brief overview of ETX, and analyze its suitability as a location estimation parameter. In Section 4, we discuss some localization algorithms used to obtain our results. We present experimental findings supporting our claims in Section 5. Finally, we depict in Section 6 the conclusions drawn, and our future work.

\section{Related Work}

Range-based localization techniques rely on specialized hardware (e.g., RF tags, ultrasound or infrared receivers, etc.) and extensive deployment of dedicated infrastructure solely for localization purpose $[12,13,14]$. They provide fine-grained location information, e.g., Active BAT [13] system is shown to have $2 \mathrm{~cm}$ average accuracy. On the contrary, range-free schemes $[4,5,15]$ provide much coarser accuracy but require no infrastructure for positioning system. Therefore, they find applicability in localizing inexpensive nodes in ad-hoc networks. Generally, various link layer metrics, e.g., RSS [4], ordered sequence of RSSs [15], Signal Strength Difference (SSD) [16], Signal-to-Noise Ratio (SNR), signal quality, etc. are utilized as location estimation parameters. DV-Hop [17], Amorphous [18] and Self-Configurable [19] localization are proposed mainly for ad-hoc networks that provide coarse-level accuracy. They use number of hops (a network layer metric) to reach a node as an indication of its distance from it. To our knowledge, no work in literature has tried to utilize the network layer metric, ETX, as a location estimation parameter for ad-hoc networks so far.

There is also a third category of localization techniques which utilizes the correlation between easily measurable signal characteristics (e.g., RSS) and location. These location fingerprinting solutions try to build a positioning system on top of existing infrastructure (e.g., Wi-Fi networks) $[20,21,16]$. Some locationdependent signal parameters (e.g., RSS) are collected at a number of locations as location fingerprints in an "offline training phase". During the "online location determination phase", the signal parameter obtained is compared with those training data to estimate the user location. This family of localization generally entails a laborious training phase which may not be attractive for the low-cost easily deployable ad-hoc networks.

\section{ETX Overview and Analysis}

\subsection{Overview of ETX}

Expected Transmission Count (ETX) is a wired/wireless link quality indication parameter. ETX predicts the number of transmissions required to deliver 
a packet over a particular link, including retransmissions. The lower the value of ETX of a link, the better the link is. The ETX of a link is calculated by utilizing the forward and reverse delivery ratios of the link. The forward delivery ratio, $\mathcal{D}_{f}$, is the measured probability that a data packet successfully arrives at the recipient; whereas the reverse delivery ratio, $\mathcal{D}_{r}$, is the measured probability that the acknowledgement is successfully received. Whenever a data packet is received by a node, it sends an ACK packet to the sender to indicate its successful reception. The sender will retransmit the packet only if it does not receive the ACK packet. This incident occurs when

- the packet sent is lost, or

- the acknowledgement sent from the recipient is lost.

Consequently, it can be seen that a successful transmission (without retransmission) of a packet incorporates both the reverse and forward delivery ratio probabilities. The expected probability that a packet is successfully received and acknowledged is, $\mathcal{D}_{f} \times \mathcal{D}_{r}$. Since each attempt to transmit a packet can be considered a Bernoulli trial, the expected number of transmission is:

$$
\mathrm{ETX}=\frac{1}{\mathcal{D}_{f} \times \mathcal{D}_{r}}
$$

In wireless networks, ETX link quality parameter has found practical application, where it is shown to be able to differentiate the high-throughput links from the lossy ones. The routing protocols (e.g., OLSR) of wireless ad-hoc networks make use of this metric in order to choose efficient routes. The ETX of a route is the sum of the ETX for each link in the route.

\subsection{ETX as a measure of proximity}

Proximity to an anchor node (i.e., node with known location) feature has been widely used in order to localize inexpensive sensors in a cost-effective way (i.e., without any infrastructure specifically deployed for localization). Centroid [4] uses the number of samples collected from an anchor node within a certain time period to indicate whether it is close to the anchor. Ecolocation [15] utilizes the signal strength measurements perceived by a node from two anchors, and picks the one with the stronger signal to be closer than the other. Based on some simplistic assumptions, we show that ETX can also be shown as a proximity indicator relative to an anchor node, and thereby, could be utilized as a location estimation parameter.

Suppose a node's communication range is, $R$, and the probability of a packet loss is, $p$. Subsequently, the probability that a packet is successfully received and acknowledged becomes, $(1-p)^{2}$.

Consider an example scenario where anchor node $B$ is within the communication range of target node $A$. The ETX of the link $A B$ can be denoted as,

$$
\operatorname{ETX}_{A B}=\frac{1}{(1-p)^{2}} \text {. }
$$


Consider another anchor node $C$ where it is inside the communication range, $R$, of node $B$ but outside the communication range of $A$. This implies,

$$
d_{A B}<d_{A C}
$$

where $d_{A B}$ and $d_{A C}$ represent the distances of node $A$ from $B$ and $C$, respectively. The ETX of the link $B C$ can be formulated in a similar way as (2),

$$
\operatorname{ETX}_{B C}=\frac{1}{(1-p)^{2}}
$$

From the definition of ETX for a route, and utilizing (2) and (4), the ETX of the link $A C$ ( $C$ is outside the communication range of $A$ ) can be written as,

$$
\operatorname{ETX}_{A C}=\operatorname{ETX}_{A B}+\operatorname{ETX}_{B C}=\frac{2}{(1-p)^{2}} .
$$

Combining (2) and (5), we obtain,

$$
\operatorname{ETX}_{A B}<\operatorname{ETX}_{A C}
$$

For two nodes, $B$ and $C$, that are inside and outside of $A$ 's communication range, respectively, both the constraints (3) and (6) are always satisfied. Therefore, ETX parameter could be used as a proximity measure for a multi-hop ad-hoc networks. Our analysis only verifies this considering two anchors $(B$ and $C)$ that are neighbors to each other. The analysis can easily be extended in a similar way for any two anchors that are separated by multiple hops. Furthermore, (3) and (6) could be merged into the following constraint:

$$
\operatorname{ETX}_{A B}<\operatorname{ETX}_{A C} \Rightarrow d_{A B}<d_{A C} .
$$

Note that, (7) is presented in the above form to depict ETX as a proximity indication parameter. However, (3) and (6) could actually be combined into the constraint, $\operatorname{ETX}_{A B}<\operatorname{ETX}_{A C} \Leftrightarrow d_{A B}<d_{A C}$ since for our particular scenario, $d_{A B}<d_{A C} \Rightarrow \operatorname{ETX}_{A B}<\operatorname{ETX}_{A C}$ as well.

Similar analysis holds if there is no packet loss, i.e., the communication channel is perfect $(p=0)$. In the analysis above, we consider two anchors where one is within the communication range of the target node, while the other one is not. In a practical scenario, both the anchors might be multiple hops away from the target node, or they both could be within the communication range of it. We consider both these scenarios in the following.

3.2.1 Anchors Multiple Hops Away: Here, we derive the constraint (7) if both anchors are multiple hops away from the target node. Suppose the nodes, $B$ and $C$ are $m$ and $n$ hops away from node $A$, respectively, where $m<n$. For simplicity of our analysis, we consider any node within the communication range, $R$, of the target node to be $R$ distances away. In other words, all nodes within $R$ are considered to be the same distance away from the target node. Subsequently, 
the nodes with the same hop count (i.e, $k$ ) turn out to be similar distances (i.e., $k R$ ) away from the target node. This interpretation holds due to our simplistic channel model which states that any node within the communication range, $R$, is associated with the same packet loss probability, $p$. So, nodes $B$ and $C$ are $m R$ and $n R$ distances away from node $A$, respectively, thereby satisfying (3) since $m R<n R$. The ETX of the links $A B$ and $A C$ can be shown as, $\operatorname{ETX}_{A B}=\frac{m}{(1-p)^{2}}$, and $\operatorname{ETX}_{A C}=\frac{n}{(1-p)^{2}}$, respectively, yielding $\operatorname{ETX}_{A B}<\operatorname{ETX}_{A C}$ since $m<n$. Consequently, the constraint (7) is satisfied. The analysis in the next section differentiates between any two anchors that are same number of hops away from the target node (i.e., $m=n$ ) but might be at different distances from it.

3.2.2 Anchors Within Same Communication Range: In this section, we consider a generic scenario where both the anchors, $B$ and $C$ are within range, $k R(k \geq 1)$, of the target node $A$. However, their distances from the target node are not the same. Note that, $k=1$ indicates the setting where both the anchors are within the communication range, $R$, of node $A$.

For this particular scenario, we drop our assumption that the channel is characterized by a constant packet loss probability, $p$. However, we assume same bandwidth and equal spectral efficiency for all the nodes within the range, $k R$. If the noise is flat, then the packet loss (i.e., bit error rate) probability between nodes $A \& B$, and $A \& C$, follows the relationship, $p_{\mathrm{AB}} \propto \frac{1}{\mathrm{RSS}_{\mathrm{AB}}}$, and $p_{\mathrm{AC}} \propto$ $\frac{1}{\operatorname{RSS}_{\mathrm{AC}}}$, respectively. From path-loss model of RF propagation [3], we obtain, $\operatorname{RSS}_{\mathrm{AB}} \propto\left(\frac{1}{d_{\mathrm{AB}}}\right)^{\beta}$, and $\operatorname{RSS}_{\mathrm{AC}} \propto\left(\frac{1}{d_{\mathrm{AC}}}\right)^{\beta}$, where $\beta$ is the path-loss exponent. If $d_{\mathrm{AB}}<d_{\mathrm{AC}}$, and the proportionality constant is the same, then, $\mathrm{RSS}_{\mathrm{AB}}>$ $\mathrm{RSS}_{\mathrm{AC}}$. Subsequently, we obtain, $p_{\mathrm{AB}}<p_{\mathrm{AC}}$ which yields $\mathrm{ETX}_{\mathrm{AB}}<\mathrm{ETX}_{\mathrm{AC}}$. Therefore, the constraint (7) is satisfied.

\section{Localization Algorithms}

In this section, we briefly discuss the range-free localization algorithms that we have utilized in order to obtain our results.

\subsection{Centroid}

The Centroid scheme [4] defines a connectivity metric which indicates the closeness of a node to an anchor. During a certain time interval, all the anchors send a predefined number of beacons. The connectivity metric is defined as the number of beacons received by the node from a particular anchor to the number of beacons sent by it during a time interval. The final location estimate is the centroid of all the anchors for which, the connectivity metric is above a certain threshold. In our implementation, all the anchors are configured in similar way so that we know the number of beacons (RSS) or messages (ETX) sent by them during a particular time interval. We just capture the beacons/messages at the target node, and compare with the threshold to decide whether it is connected to a particular anchor or not. 


\subsection{Nearest Neighbor}

Nearest Neighbor (NN) algorithm [22] returns the anchor's location from which the node receives the strongest signal (RSS) or perceives the best link quality metric (ETX). K-nearest neighbor $(\mathrm{KNN})$ is a variant of the basic NN algorithm where $K$ anchors' location entries are returned instead of returning only the best match. The final location estimate is obtained by averaging the coordinates of the $K$ anchors' locations found. The value of $K$ has usually been chosen empirically in the literature. In our implementation, we have chosen $K$ to be 3 .

\subsection{Ecolocation}

Ecolocation [15] uses ordered sequence of RSS measurements rather than the absolute RSSs. If $P\left(d_{i}\right)$ and $P\left(d_{j}\right)$ denote the RSSs from anchor $i$ and anchor ${ }_{j}$, which are at distances $d_{i}$ and $d_{j}$ from the node, respectively, then a constraint of the sequence is defined as,

$$
P\left(d_{i}\right)>P\left(d_{j}\right) \Rightarrow d_{i}<d_{j}
$$

First, the constraint set for each grid point of the localization area is calculated using the RHS of (8). During location determination phase, the ordered sequence of RSSs collected from the anchors is translated into the ordered sequence of distances using (8), and subsequently matched against the constraint set of each grid point calculated beforehand. The centroid of the grid points where the maximum number of constraints are matched is returned as the location estimate. Ecolocation can easily be adopted for ETX based localization as well using the constraint (7) derived in Section 3.2.

The discussion on fingerprint based localization algorithms, e.g., Bayesian Inference [21] and KNN in Signal Space [20] has been omitted for brevity.

\section{Experimental Study}

We first describe our experimental testbed and data collection procedure in Section 5.1. Then, we provide our results and findings in Section 5.2.

\subsection{Testbed Setup and Data Collection}

The experimental testbed is located inside our research lab which spans over an area of approximately $663 \mathrm{~m}^{2}$. We have used six Mini Computer MicroClient Jr. (200 MHz thin client) to serve as anchors for our experiments. The choice of such devices is derived from our motivation to accommodate inexpensive devices or sensors. The locations of these anchors are shown in Fig. 1, marked as stars. The grids where data are collected are indicated by crosses. All our mini PCs run Puppy Linux 4.3.1 Linux distribution while our target node (an Asus Eee PC 901) runs Ubuntu 9.10. The target node has a Linksys WUSB54GC 


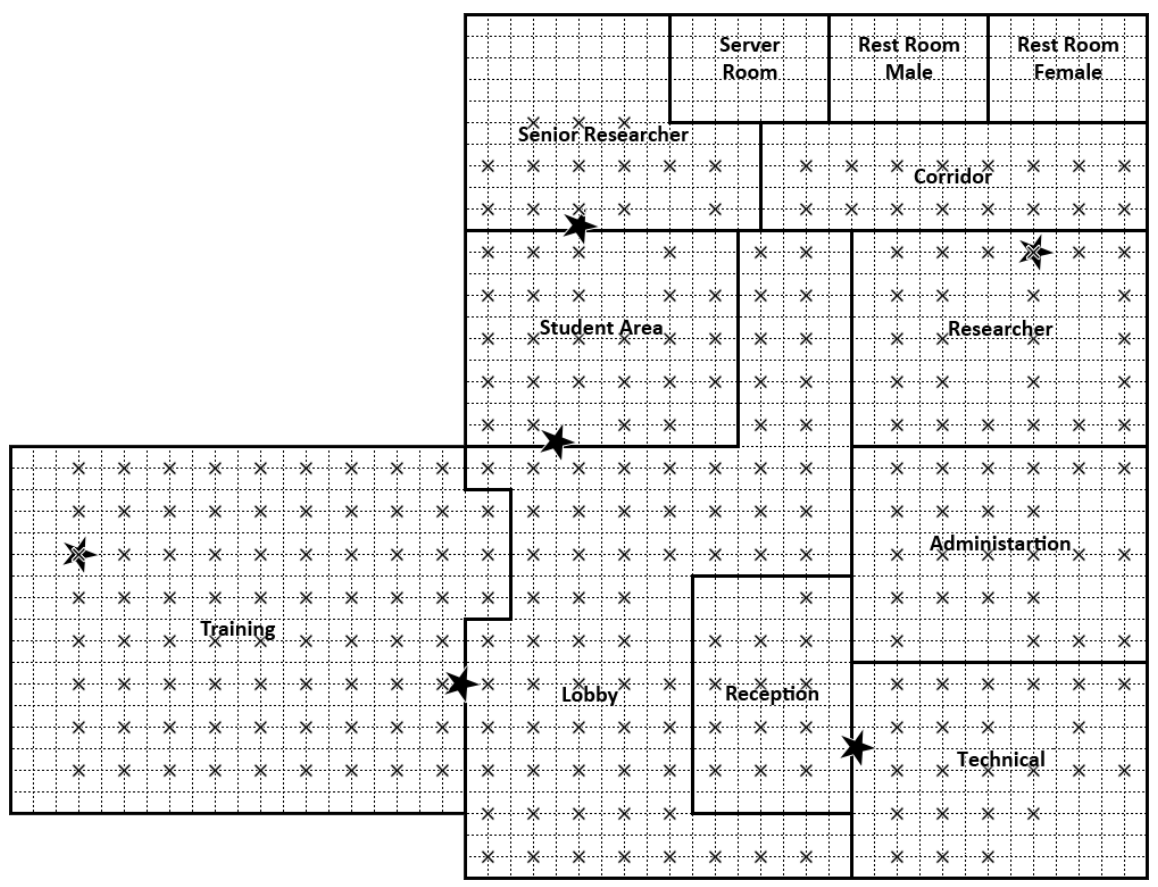

Fig. 1. Our experimental testbed inside our research lab - the six anchors are marked as stars, and all the data collection points are marked as crosses.

WLAN adapter attached to it while each mini PC is installed with Realtek 8100B chip with an external antenna. All of them create an ad-hoc network, and run OLSR [23].

In our testbed, there are 281 training points or grids, marked as crosses in Fig. 1 where we collect both RSS and ETX measurements. For RSS, we have utilized "tcpdump" packet analyzer to capture the signal strength information at the target node stationed at a particular grid. We first put the node's NIC into monitor mode, and then run tcpdump where it snoops all the 802.11 packets from the air, and only retrieves the required RSS information from our desired anchors. For ETX, we consult the OLSR routing table at the target node periodically, and retrieve the ETX information only for the anchors. We have actually built a plugin for the OLSR daemon based on ETX which provides the location information of the node in our lab.

\subsection{Experimental Results and Findings}

5.2.1 Simplistic Localization Algorithms for Ad-hoc Networks: We present the results of the localization algorithms discussed in Section 4 that are commonly applied for ad-hoc networks. We feed both RSS and ETX as location estimation parameter into the algorithms. We calculate the deviations (in meters) between actual and predicted locations for our 281 measurements, and 


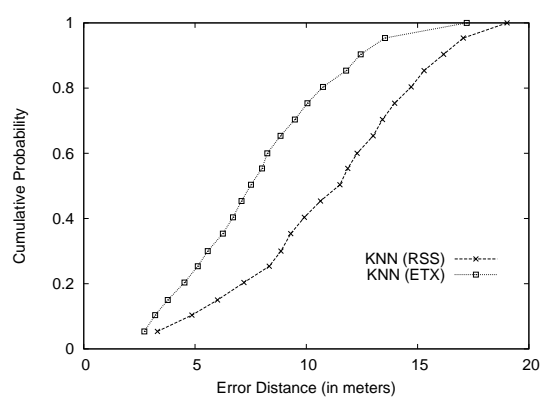

(a) K-Nearest Neighbor (KNN).

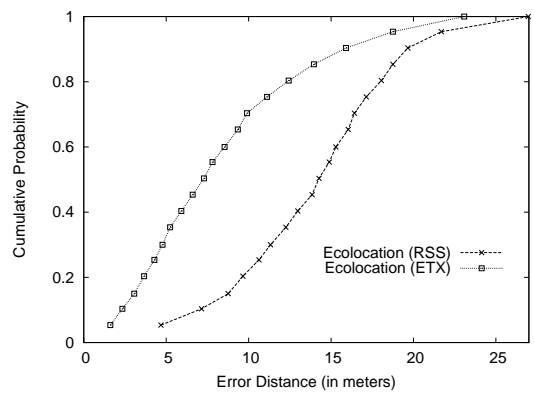

(c) Ecolocation.

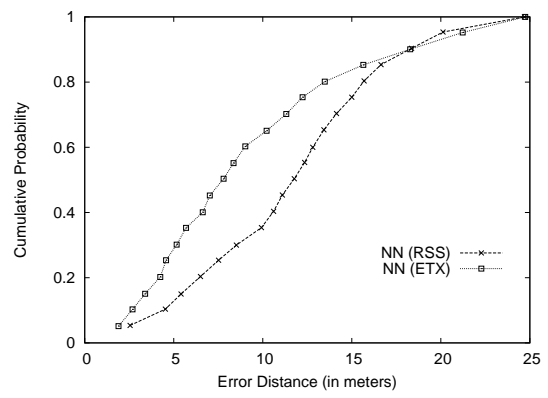

(b) Nearest Neighbor (NN).

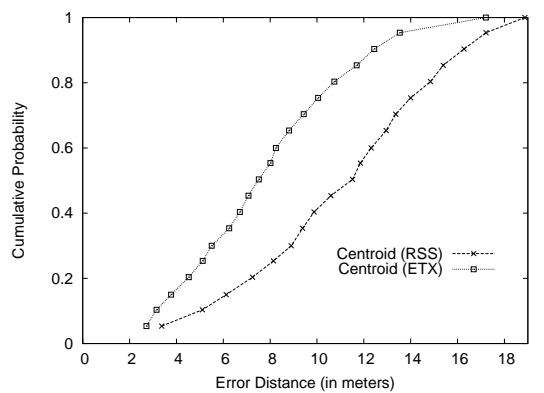

(d) Centroid.

Fig. 2. Comparison of error performance for various simplistic localization algorithms seen in ad-hoc networks using both RSS and ETX.

draw the graphs of Fig. 2. The graphs reveal the percentage of locations (Y-axis) predicted correctly within a specific error distance (X-axis).

As can be seen from the graphs of all algorithms, ETX generally outperforms its counterpart RSS. These simplistic algorithms for ad-hoc networks is built upon the idea of "proximity measure" to a node with known location. ETX is shown to be an efficient proximity measure in the analysis of Section 3.2. Our experimental results here verify that. On the contrary, RSS is affected by reflection, diffraction and multi-path effect [3] which compels it to correlate poorly with distance. The performance gain of our ETX based Ecolocation compared to RSS based Ecolocation [15] is quite significant as depicted in Fig. 2(c). This is due to the fact that our ETX based constraint (7) follows quite nicely under some simplistic assumptions. On the contrary, RSS measurements generally do not represent distances accurately in the real world. Therefore, uncertainties could arise while using (8) as discussed in [15].

The numerical values (averages) of the experiments are listed in Table 1. It can be noted that all the accuracies reported by these simplistic algorithms are quite coarse which is in accordance with the findings of existing literature $[4,5]$.

5.2.2 Fingerprint-based Algorithms: In the previous section, we show ETX to perform better than RSS considering some simple localization algorithms 


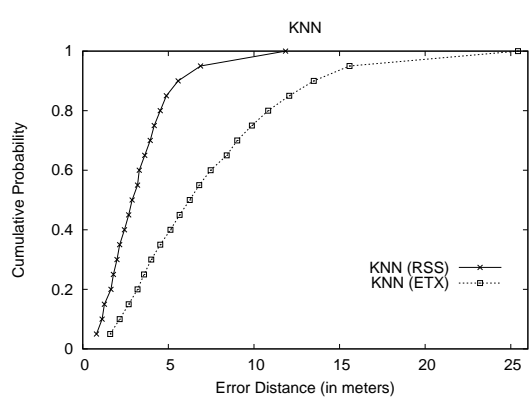

(a) KNN in Signal Space.

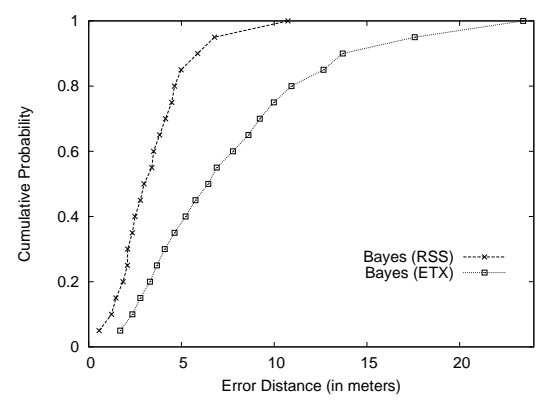

(b) Bayesian Inference.

Fig. 3. Comparison of error performance for fingerprint-based localization algorithms using both RSS and ETX.

designed specifically for wireless ad-hoc networks. Here, we investigate both RSS and ETX's performance regarding fingerprint-based algorithms even though such techniques may not be suitable for ad-hoc networks because of the burden of their deployment. Since our goal is to investigate whether ETX could be utilized as a location estimation parameter similar to RSS irrespective of the algorithms used, we consider these fingerprinting solutions for completeness.

For this experiment, in each trial, 100 testing samples are selected randomly from the 281 data points, and the rest 181 samples are kept as training database for fingerprinting algorithms [20, 21]. We repeat this procedure for 101 times to obtain the graph of Fig. 3(a) and 3(b), and the averages with $95 \%$ confidence interval as shown in Table 2. The confidence interval is calculated using the results of 101 runs as a vindication that our results are not biased towards a particular separation of training and testing set.

From Fig. 3(a) and 3(b), it is evident that RSS based fingerprinting solutions comfortably outperforms the ETX based ones. The fingerprinting techniques involve collecting signal parameters (e.g., RSS) at places of interests, and subsequently building a radio-map [20] by correlating the signal parameters with locations. The signal parameters measured during the location determination phase is compared with the data of the radio-map collected beforehand, which is just pattern matching in signal space. Although RSS is reported to vary with many factors [15, 24], fingerprinting techniques based on RSS is quite popular in existing literature $[20,21,16]$ which provide reasonable average accuracy $(2 \sim 4 \mathrm{~m})$. Our results here show similar trend (see Table 2). Apart form environmental surroundings, time of the day, etc., which are the common influencing factors for both ETX and RSS, ETX is also affected significantly by congestion, node density in ad-hoc networks, etc. A more detailed analysis can be found in [25]. These factors make ETX a poor candidate for location fingerprint since ETX stored at some place beforehand may hardly follow the same pattern at the same place later even though the proximity constraint (7) is satisfied in both occasions. Therefore, ETX based fingerprinting techniques is quite inferior to 
Table 1. Average Errors (in meters) of the Simplistic Localization Algorithms for Ad-hoc Networks for the Experiments Conducted in Section 5.2.1

\begin{tabular}{c|c|c|c|c} 
& KNN & NN & Ecolocation & Centroid \\
\hline RSS & 10.83 & 11.41 & 13.78 & 10.87 \\
\hline ETX & 7.67 & 9.08 & 8.12 & 7.66 \\
\hline
\end{tabular}

Table 2. Average Errors with Confidence Interval (in meters) of the Fingerprint-based Localization Algorithms for the Experiments Conducted in Section 5.2.2

\begin{tabular}{c|c|c} 
& KNN & Bayes \\
\hline RSS & $3.19 \pm 0.03$ & $3.28 \pm 0.02$ \\
\hline ETX & $7.11 \pm 0.07$ & $7.14 \pm 0.07$ \\
\hline
\end{tabular}

RSS based ones (see Fig. 3(a) and 3(b)) although its performance is slightly improved compared to the algorithms of Section 5.2.1.

\section{Conclusion}

In this paper, we propose an ETX based positioning system for wireless adhoc networks. We analytically show that ETX could be utilized as a proximity indication parameter and thereby, it can easily be adopted for the localization algorithms designed for wireless ad-hoc networks comprising of inexpensive nodes. Such ETX based systems are shown to outperform the RSS based ones in our experiments. However, ETX performed poorly compared to RSS in case of fingerprint based algorithms that are not suitable for wireless ad-hoc networks because of its exhaustive data collection procedure. In future, we plan to extend our work by considering other existing localization algorithms in addition to the popular ones that are considered here. We shall also investigate our ETX based system's performance in an anchor-free scenario utilizing collaborative localization approach [26].

\section{Acknowledgement}

This work has been supported by Intelligent Transportation System Cluster of the NSTDA, Thailand and the THNIC Foundation.

\section{References}

1. J. F. Kurose and K. W. Ross, Computer Networking: A Top-Down Approach. Addison-Wesley Publishing Company, 2009.

2. I. F. Akyildiz, W. Su, Y. Sankarasubramaniam, and E. Cayirci, "A survey on sensor networks," IEEE Commun. Mag., vol. 40, no. 8, pp. 102-114, 2002.

3. T. S. Rappaport, Wireless Communications - Principles and Practice. Prentice Hall, 1996.

4. N. Bulusu, J. Heidemann, and D. Estrin, "GPS-less low-cost outdoor localization for very small devices," IEEE Personal Communications Magazine, vol. 7, no. 5, pp. 28-34, 2000.

5. T. He, C. Huang, B. M. Blum, J. A. Stankovic, and T. Abdelzaher, "Range-free localization schemes in large scale sensor networks," in Proc. ACM/IEEE Mobicom'03, 2003, pp. 81-95. 
6. J. Bardwell, "A discussion clarifying often-misused 802.11 WLAN terminologies." [Online]. Available: http://www.connect802.com/download/techpubs/2004/you_ believe_D100201.pdf

7. D. B. Johnson, "Routing in ad hoc networks of mobile hosts," in Proc. of the IEEE Workshop on Mobile Computing Systems and Applications, 1994, pp. 158-163.

8. C. E. Perkins and P. Bhagwat, "Highly dynamic destination-sequenced distancevector routing (dsdv) for mobile computers," in Proc. ACM SIGCOMM Conference (SIGCOMM 94), 1993, pp. 234-244.

9. P. Jacquet, P. Mühlethaler, T. Clausen, A. Laouiti, A. Qayyum, and L. Viennot, "Optimized link state routing protocol for ad hoc networks," in Proc. of IEEE INMIC, 2001, pp. 62-68.

10. C. E. Perkins and E. M. Royer, "Ad-hoc on-demand distance vector routing," in Proc. of the 2nd IEEE Workshop on Mobile Computing Systems and Applications, 1997, pp. 90-100.

11. D. S. J. De Couto, D. Aguayo, J. Bicket, and R. Morris, "A high-throughput path metric for multi-hop wireless routing," in Proc of MobiCom'03, New York, NY, USA, 2003, pp. 134-146.

12. R. Want, A. Hopper, V. Falcão, and J. Gibbons, "The active badge location system," ACM Trans. on Information Systems, vol. 10, no. 1, pp. 91-102, Jan. 1992.

13. A. Ward, A. Jones, and A. Hopper, "A new location technique for the active office," IEEE Personal Communications, vol. 4, no. 5, pp. 42-47, Oct. 1997.

14. N. Priyantha, A. Chakraborty, and H. Balakrishnan, "The Cricket location-support system," in Proc. ACM MobiCom'00, Boston, MA, Aug. 2000, pp. 32-43.

15. K. Yedavalli, B. Krishnamachari, S. Ravula, and B. Srinivasan, "Ecolocation: a sequence based technique for RF localization in wireless sensor networks," in Proc. ISPN'05, Apr. 2005.

16. M. Hossain, H. Nguyen Van, Y. Jin and W.-S. Soh, "Indoor localization using multiple wireless technologies," in Proc. IEEE MASS, Pisa, Italy, Oct. 2007. [Online]. Available: http://www.ece.nus.edu.sg/stfpage/elesohws/mass07.pdf

17. D. Nicolescu and B. Nath, "DV based positioning in ad hoc networks," Journal of Telecommunications Systems, 2003.

18. R. Nagpal, "Organizing a global coordinate system from local information on an amorphous computer," MIT A.I. Laboratory, Tech. Rep. A.I. Memo 1666, 1999.

19. H. Wu, C. Wang, and N.-F. Tzeng, "Novel self-configurable positioning technique for multi-hop wireless networks," IEEE/ACM Transactions on Networking, vol. 13, no. 3, pp. 609-621, 2005.

20. P. Bahl and V. N. Padmanabhan, "RADAR: An in-building RF-based user location and tracking system," in Proc. IEEE INFOCOM, Tel Aviv, 2000, pp. 775-784.

21. M. A. Youssef, A. Agrawala, and A. U. Shankar, "WLAN location determination via clustering and probability distributions," in Proc. IEEE PERCOM'03, 2003.

22. A. K. Jain, R. Duin, and J. Mao, "Statistical pattern recognition: A review," IEEE Transactions on Pattern Analysis and Machine Intelligence, vol. 22, pp. 4-37, 2000.

23. olsrd, "An adhoc wireless mesh routing daemon." [Online]. Available: http://www.olsr.org

24. K. Kaemarungsi and P. Krishnamurthy, "Properties of indoor received signal strength for WLAN location fingerprinting," in Proc. MobiQuitous'04, San Diego, CA, 2004, pp. 14-23.

25. S. M. Das, H. Pucha, K. Papagiannaki, and Y. C. Hu, "Studying wireless routing link metric dynamics," in Proc. of IMC'07, NY, USA, 2007, pp. 327-332.

26. N. B. Priyantha, H. Balakrishnan, E. Demaine, and S. Teller, "Anchor-free distributed localization in sensor networks," in Proc. of SenSys'03, 2003, pp. 340-341. 